\title{
Mejoramiento del servicio al cliente a los estudiantes matriculados en una universidad a distancia de la ciudad de Cali \\ Diagnóstico inicial
}

\author{
Orlando Montenegro Romero ${ }^{1}$ \\ Suria Alexandra Melendez Martine ${ }^{2}$ \\ Jennifer Magnolia Ortiz Lozada ${ }^{3}$
}

\section{Resumen}

En el presente trabajo se realiza un diagnóstico del nivel del servicio que se presta en una universidad a distancia de la ciudad de Cali, a partir del cual se genera las propuestas de acción que tienen como objetivos generar estrategias de fidelización, retención y promoción en los estudiantes, teniendo en cuenta que el proceso comercial en las etapas de ventas y Marketing genera un valor inicial para el cliente, que en el caso de la esta universidad es el estudiante, el cual debe tener valores diferenciales que debe percibir éste cuando compra y usa los servicios de la universidad, para este logro se deben desarrollar estrategias y tácticas de servicio al cliente que permitan el logro de impactos importantes en la fidelización del target del mercado que se atiende, fortaleciendo el plan estratégico y la postura competitiva de la universidad en el mercado en el cual compite.

\footnotetext{
${ }^{1}$ Psicólogo, Especialista en Educación, Cultura y Política, Estudiante Maestría Intervención Psicosocial Universidad, docente Universidad Nacional Abierta y a Distancia - UNAD, Grupo Subjetividades y Sujetos Colectivos orlando.montenegro@unad.edu.co

${ }^{2}$ Ingeniera Industrial Universidad Autónoma de Occidente, Estudiante Psicología Universidad Nacional Abierta y a Distancia - UNAD

${ }^{3}$ Estudiante Psicología Universidad Nacional Abierta y a Distancia - UNAD
} 
El fomento y la promoción de la competencia de orientación al servicio en los colaboradores de la institución se hace imperativo de manera que ellos se conviertan en un sistema de apoyo para que la estrategia comercial se ponga en marcha de manera efectiva.

La metodología utilizada se enmarca dentro del paradigma cualitativo utilizando diferentes técnicas de recolección de la información como la observación, los grupos focales la prueba de algodón y la entrevista abierta y cuyos resultados proveerán de información suficiente para proponer acciones de acompañamiento que mejoren el servicio que presta la institución a sus estudiantes.

Palabras Clave: Servicio al cliente, educación virtual, retención, fidelización.

\section{Introducción}

La fidelización y la retención estudiantil es un tema relevante en la Universidad a distancia ya que a pesar de que año tras año cientos de estudiantes dan inicio a sus carreras, un alto porcentaje deserta, fenómeno que amerita una revisión profunda, motivo por el cual en nuestro caso particular a través de este proyecto se pretende dar una contribución significativa a la disminución de la deserción en la Unidad Regional de la ciudad de Cali; fin que contempla como una de las tareas a direccionar los esfuerzos para fortalecer la relación de los estudiantes y potenciar el compromiso individual de los que han logrado iniciar sus estudios, para que logren dar término efectivo a su proceso educativo.

Uno de los factores más importantes para que los estudiantes permanezcan y lleguen a la universidad, son los estudiantes que egresan de la misma y logran los objetivos que se propusieron cuando realizaron su proyecto de vida, permitiéndoles posicionarse en el mercado laboral, lo cual los convierte en los mejores promotores de la institución, pues 
cuentan con la opción de continuar sus estudios pos-gradúales, al igual que recomendar o referir a otras la institución.

\section{Problema}

Los estudiantes de la universidad a distancia en la sede de Cali, requieren de una atención acorde a la certificación ISO 9001-2008, a la Política y Objetivos del Sistema Integrado de Gestión de calidad de la universidad, al programa de Inducción y acogida de la universidad y a las funciones de la Vicerrectoría de servicios a aspirantes, estudiantes y egresados, de manera que puedan lograr los objetivos académicos que cada uno de estos se han planteado en su proyecto de vida. Teniendo en cuenta lo anterior en las instalaciones de la universidad en la ciudad de Cali, se requiere prestar un adecuado servicio a los estudiantes, que permita la fidelización de estos a la institución, lo que redundará en la permanencia y retención.

Ahora bien, consciente del impacto que tiene el servicio al cliente en los estudiantes, y con el fin de responder a las exigencias actuales del mercado y mejorar el nivel de la atención, se decide diseñar una propuesta de intervención, la cual les permitirá reducir y prevenir los impactos que se genera la prestación de un inadecuado servicio y a su vez generar oportunidades de mejora.

Con lo anterior se plantea la siguiente pregunta: ¿Cómo mejorar el nivel de la atención que se presta a los estudiantes de las diferentes carreras que se encuentran matriculados en la Universidad en la Regional de la ciudad de Cali en el periodo 2017-16-1 y 2 ? 


\section{Marco Teórico y Conceptual}

Para el caso particular de la sede de la ciudad de Cali, fortalecer la relación con los estudiantes y clientes potenciales, es un propósito que se logra haciendo énfasis en el trato cordial y esmerado, esto consolida un clima institucional positivo que es percibido fácilmente, que genera sentimientos de bienestar, permanencia, fortalece la identidad institucional, hace que los procesos eleven su eficacia y significado. Como lo indica Torres (2012):

(...) el clima institucional es un factor que puede fomentar o inhibir el crecimiento social e intelectual de un estudiante, (...). Las investigaciones coinciden en que un ambiente institucional adecuado y la integración al campus son importantes para retener a los estudiantes hasta su graduación. Además, se debe tener en cuenta que el clima del campus influye en las experiencias académicas y sociales de los estudiantes de pregrado en la universidad. (p.57)

Es decir, que favorecer el clima institucional eleva la calidad del servicio, mejora el trabajo a nivel interno haciéndose más ameno y eficaz, asegura procesos académicos más estables, fortalece el sentido de pertenencia e identidad, permitiendo el desarrollo de futuros profesionales con un nivel de satisfacción importante que se convierte en la mejor referencia de la institución universitaria.

Cada individuo asume una perspectiva propia referente a la experiencia que está viviendo, como lo indica Jiménez, (citado por La Universidad de Cantabria) sobre la Teoría de la Acción Razonada de Fishbein y Ajzen, desde la cual se deduce que hay una influencia marcada de las creencias y las actitudes en los estudiantes y la manera en que afrontan las 
diferentes situaciones alrededor de su ciclo educativo, esta información que se procesa a nivel individual se convierte en el pilar de la decisión sobre continuar o no en la Universidad. Al entender que un momento de contacto va más allá del “aquí y ahora”, es posible ser más consciente sobre el poder de influencia que se tiene en los estudiantes y la calidad de su proceso de formación, por ello se hace vital ver más allá de la oferta, ver más allá de las bondades del servicio y el afán de promoverlo, y centrarse en construir relaciones. La Universidad, cuenta con varios reconocimientos de calidad a nivel nacional e internacional, uno de ellos es la ISO 9001, logro que es motivo importante para fortalecer con premura el manejo de los procesos internos en la Sede Regional de Cali, que permita alcanzar resultados mayores a nivel social y económico. Como Organización, la Sede regional de Cali, funciona a través de las diferentes áreas y/o dependencias para la ejecución de sus tareas, estructura que la convierte en todo un sistema, como lo indica Velásquez (2007):

La organización es pues un sistema orgánico (...) socio-técnico abierto, el cual posee, relaciones de entradas (insumos), salidas (productos) y retroalimentación o ciclos de retorno para modificar el propio sistema, en estructura, operación, función o propósito, permitiendo su permanencia en el tiempo, además de procesos internos de readaptación, construcción y auto reparación, que le permiten interrelacionarse adecuadamente con un entorno. (p.132)

Es decir que una revisión a sus procesos requiere una mirada detallada para lograr un análisis de la situación más certero, un accionar efectivo que permita mejoras en la dinámica general de la institución. En este caso como lo indica Velásquez (2007) "sistema y organización debe centrase en su principal función, dar identidad para posibilitar la diferencia y por tanto mejorar la percepción del observador” (p.131). La solidez es el 
resultado de esa relación clara y armoniosa por la que se debe impulsar todos los esfuerzos, que son los que dan la imagen final en la que los clientes, en este caso los estudiantes se van a fijar, es parte de ese recuerdo con el que grabaran en su mente la experiencia, como lo dice Luhmann (citado por Velásquez, 2007): Las organizaciones son esos sistemas sociales que se permiten tratar al comportamiento humano tal como si fuera un decidir. La realidad social del decidir en organizaciones es interpretada por consiguiente como la sola aceptación o suposición o sugestión de los participantes en el sistema (p.138).

Así se evidencia que ese "decidir" es el resultado de la suma de todos los elementos que circundan en el sistema, por lo que hay que estar alerta a esa dinámica continua que se suscita en cada área de la estructura, debido a que una partícula alterada afecta al resto del Macrosistema, como lo indican Peter Senge en La V disciplina y Joseph O’Connor y Ian McDermott en su libro titulado Introducción al pensamiento sistémico de momentos esperados (Velásquez,2007) "El pensamiento sistémico es un instrumento fundamental para auto-guiarse y dirigir a otros con eficacia. El pensamiento sistémico es una reflexión en círculos más que en línea recta.” (p.142).

Es por ello que su alteración afecta directamente a las otras, en el caso particular del servicio al cliente, el ideal es prestar y recibir el mejor servicio, pero las fallas que puedan permear los procesos ponen en riesgo tales ideales, tal disposición, punto en el que se pone a prueba la fortaleza de la organización en el sentido del servicio. La dependencia de unos y otros niveles es continua, la autosuficiencia no cabe dentro de la dinámica organizacional, así que una estructura que quiere funcionar, pero también ser amigable, cordial con sus clientes (Macrosistema) ha de aprender a serlo desde su interior (microsistemas), ha de ser un trabajo coordinado para lograr la estabilidad. 
Es importante resaltar, la visión clara que se puede obtener de la organización desde el enfoque sistémico, ya que al revisar cada proceso y también la suma de todos ellos, es posible obtener una visión global y particular de todo el sistema, lo cual llevará a que se identifiquen las situaciones críticas y a su vez las opciones de mejora; lo que no es posible desde un análisis solamente cuantitativo, que es el camino usualmente transitado en las organizaciones, el cual puede arrojar porcentajes y un estudio minucioso racional, pero poco asertivo en cuanto a condicionantes que pueden estar presentes en el entorno y afectar los procesos, como lo indica Bonilla (2010):

Tales fallas y deficiencias fundamentalmente obedecen a la falta de una visión conjunta de la organización, en distintos planos de intervención en y con su entorno (interno y externo) y en la cultura de la organización prevalece como eje rector el enfoque racionalista sobre el sistémico. (Pag. 389).

El aporte desde el punto de vista sistémico es ideal para el mejoramiento del servicio al cliente en las organizaciones, pues su aplicación evidencia las diferencias, posibilidades, fallas y deficiencias en las diferentes áreas, permitiendo realizar acciones puntuales y centradas en momentos críticos del proceso, que llevan a la aplicación eficaz de acciones que mejoren la operación y el desarrollo de cada proceso, y por ende se obtengan mejores resultados a nivel global.

Para una mejor compresión de la situación planteada se exponen los principales conceptos a saber: Los clientes son la razón de ser de la organización, ya que como se indica en la ISO 9001 (2013), el concepto de cliente se refiere a cliente como “Organización o persona que recibe un producto", es decir es a quien debe ir dirigido todo el esfuerzo de la institución, es en ese momento de entrega donde se pone en juego la 
estabilidad presente y futura de la organización, ya que el cliente espera recibir el producto o servicio de forma satisfactoria, por lo menos esa es la idea que le ha movido a escoger esa empresa y no otra; por lo tanto no cubrir esas expectativas representa un riesgo alto para la institución prestadora del servicio. Cabe notar que la Organización ISO, da claridad sobre los dos tipos de clientes que hacen parte de la empresa, definidos así:

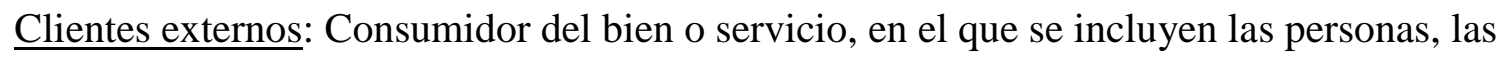
empresas o el mercado en general y que tiene la característica de ser independiente a la organización. Es el destinatario del producto o servicio producido.

Clientes internos: Representan el área, departamento, sección, personal, etc. que emplean o consumen los productos obtenidos, pero con la característica particular de pertenecer al conjunto de la organización. De este modo, dentro de la organización todos se convierten en clientes y proveedores a la vez. Por ser el cliente la razón de ser de la organización, ha de prestar notable interés en su forma de ser, para el cual prima la satisfacción de necesidades, orden en el cual dará mayor interés a una cosa que a otra, esto es posible revisando la Teoría de las Necesidades de Abrahan Maslow, (citado por Elizalde A., Martí M., Martínez F., 2006) en la cual el autor refiere que una persona está motivada cuando siente deseo, anhelo, voluntad, ansia o carencia. La motivación estaría compuesta por diferentes niveles, cuya base jerárquica de necesidad varía en cuanto al grado de potencia del deseo, anhelo, etc. Se puede inferir por lo tanto que el asiento base de la atención al cliente parte del conocimiento de la organización respecto a sus clientes internos y externos. Los clientes como individuos son seres emocionales, tienen un diseño basado en sensaciones expresadas en cada momento de manera espontánea, esas emociones definidas por Lawler (citado por Bericat, 2012), "como estados evaluativos, sean positivos o 
negativos, relativamente breves, que tienen elementos fisiológicos, neurológicos y cognitivos" (p.2). por lo que el papel es fundamental en cada una de las etapas de la vida, es así que se pueden considerar las emociones como el hilo conductor del camino, pues trazan de forma determinante los espacios que le generan complacencia, los cuales son el momento idóneo donde la persona quiere estar. Por lo tanto, una placida emoción se convierte en un camino recurrente que el individuo querrá experimentar una y otra vez, es ese comportamiento individual del consumidor, que en palabras de Andreu, L. (s.f.) son el “conjunto de actividades (cognitivas, emocionales y acciones físicas) que las personas desarrollan cuando buscan, compran, evalúan, usan y disponen de los bienes con el objeto de satisfacer sus necesidades y deseos", las emociones generan comportamientos, que son la evidencia final del gusto, anhelo y o interés del sujeto, lo que se va organizando como parte de su construcción individual, por lo que se puede decir que cada persona se va construyendo a partir de una base ciertamente vulnerable, esa base son las emociones, las cuales aportan significado no solo a los momentos, sino que va generando una suma de ellos, de manera que al final se obtiene no experiencias satisfactorias aisladas, sino toda una trama llena de sentido.

Teniendo en cuenta esa construcción individual esta universidad, desde su misión y visión, plantea su propuesta institucional direccionada al desarrollo personal y social, teniendo como pilares el avance, la vanguardia, la cobertura, los valores, entre otros, conjunto de elementos que aportan de manera significativa a esa construcción individual. En el área del servicio, es importante tener claridad sobre el ciclo de servicio, que representa como lo dice Duque, E. (2005) “...ayuda a los miembros de las organizaciones a ofrecer asistencia a los clientes, permitiéndoles organizar las imágenes mentales de lo que 
ocurre” (p.66), el cual requiere no solo coherencia, sino también significado, a través del cual se supla las necesidades del cliente pero también se logre una satisfacción elevada que genere esas sensaciones de bienestar y gusto, las cuales deben ser recurrentes en cada

momentos de verdad, concepto de Albrecht (citado por Duque, E. 2005), que en palabra de Duque, E. (2005) “es cualquier situación en la que el cliente se pone en contacto con algún aspecto de la organización y obtiene una impresión sobre la calidad de su servicio” (p.66). tener en cuenta que cada contacto con el cliente, es la oportunidad de afirmarlo como parte vital de la organización, es el espacio preciso para hacerle sentir que es el centro de toda la estructura.

\section{Metodología}

Se determina que el método donde enmarcamos el diagnóstico es el del paradigma cualitativo, se conoce también como naturalista, fenomenológico y posteriormente hermenéutico. La pretensión del paradigma es comprender la realidad social, concibiendo a la misma como resultado de un proceso histórico, considerado desde la lógica y sentir de sus protagonistas.

Siendo la realidad una construcción, donde se necesita al sujeto que es contextualizado, influido por su cultura, sus relaciones sociales y el momento histórico en el que se encuentra, de manera que el conocimiento es una construcción que se comparte a partir de la relación del investigador y del objeto investigado. Colocando así a la subjetividad y la intersubjetividad como herramientas a ser utilizadas, no obstáculos en la investigación (Samaja, 1994; Briones, 2002). 
Las representaciones sociales permiten a los sujetos decodificar, interpretar, actuar en uno u otro sentido respecto a la realidad en la cual están inscritos. Dichas representaciones incluyen: “... las formas de acción, los juicios, las emociones y las valoraciones personales de las personas que en tanto configuraciones culturales y políticas permiten informarnos sobre cómo es vivida la ciudadanía, por los propios sujetos, es decir, las y los ciudadanos" (Villarroel, Brito \& De Armas, 2004, p. 181).

El propósito de este enfoque se basa de una rigurosa descripción de las situaciones, la cual debe garantizar la máxima intersubjetividad en la captación de una realidad compleja. Lo cual permite la categorización de los datos, lo cuales deben ser cogidos de manera sistemática. Se considera fundamental escoger una teoría desde el inicio del trabajo, para darle soporte a los procesos y planteamientos que se generen durante la misma.

Se escogió el enfoque cualitativo porque permite responder a la pregunta problema, a través de la consecución de los objetivos general y específicos del ejercicio de trabajo, que pretende identificar y clasificar el nivel del servicio que se presta a los estudiantes de las diferentes carreras, que se encuentran matriculados en la UDR de Cali.

\section{Análisis y Perspectivas}

El alcance de este trabajo es estructurar las herramientas que permitan mejorar el nivel de la prestación del servicio de la universidad en su sede de Cali, de manera que se logre la fidelización de los estudiantes con la institución. Para ello se utilizaron diferentes técnicas de recolección de la información las cuales fueron: la observación, los grupos focales (Focus Group), el Net Promoter Score (NPS) o Prueba del Algodón y la entrevista abierta y en profundidad de las cuales resultados que indican que los estudiantes no les 
gusta de la universidad ciertas actividades curriculares, el desempeño y atención de los tutores y la atención al estudiante en las instalaciones de la Universidad en Cali, se debe mejorar en las ofertas de posgrado, la comunicación digital sincrónica, las instalaciones, la atención por parte de los funcionarios administrativos y tutores y los horarios de atención.

Con estos resultados se pretende construir una propuesta de intervención dirigida a proponer acciones de acompañamiento que permita el cierre de brechas en el nivel del servicio que presta la Universidad en la ciudad de Cali, que como consecuencia redunde en la fidelización, retención y promoción en los estudiantes de primera y segunda matrícula.

\section{Referencias Bibliográficas}

Albrecht, K. \& Zemke, R. (1988). Gerencia del Servicio. Bogotá, Colombia: Legis Editores. Recuperado de: https://es.scribd.com/doc/134814001/gerencia-del-servicio-pdf

Alcaide, J. (2009, abril-junio). Diez mejoras urgentes en el servicio al cliente. Gestión. Recuperado de http://docplayer.es/5257789-Gestion-abr-jun-09-abril-junio-2009-edicion-trimestralissn-1999-5709-ram-charan-el-consejero-de-los-ceo.html.

Andreu, L (s.f.). Jornadas sobre tendencias en consumo y nuevas oportunidades de negocio: Las emociones en el comportamiento de compra. Universidad de Valencia. Recuperado de http://www.pateco.org/administracion/ficheros/emociones

Arango, C. (2006). La Investigación - Acción - Participativa y la Psicología. Psicología Comunitaria de la Convivencia. Universidad del Valle, (p.p. 197-215). Cali: Universidad del Valle programa Editorial.

Bericat, E. (2012). Emociones. Sociopedia. Isa. Volumen: numero. 1-13. Doi:

\section{$10.1177 / 205684601261$}


Boletín educación superior en cifras (2016) - Concentración de graduados: Cómo va la graduación y la deserción en la educación superior. Recuperado de: http://www.mineducacion.gov.co/1759/articles-359642_recurso.pdf

Boletín Educación Superior en cifras (2016). Factores Determinantes de la Deserción. Recuperado de: http://www.mineducacion.gov.co/1759/articles-358136_recurso.pdf

Boletín No. 12. Educación Superior en Cifras (2015). Una mirada a los graduados de posgrado en Colombia: características e indicadores de mercado laboral. Recuperado de: http://www.mineducacion.gov.co/1759/articles-350451_recurso_12.pdf

Boletín No. 9. Educación Superior en Cifras (2015). Permanencia y Graduación: Una apuesta por la Equidad en Educación Superior. Recuperado de: http://www.mineducacion.gov.co/1759/articles-350451_recurso_9.pdf

Bonilla, E., \& Rodríguez, P. (2000). Manejo de datos cualitativos. In B. E \& P. Rodríguez (Eds.), Más allá del dilema de los métodos. La investigación en ciencias sociales (pp. 243- 310). Bogotá, Universidad de los Andes: Grupo Editorial Norma.

Briones, G. (2002). Epistemología de las ciencias sociales. Especialización en teoría, métodos y técnicas de investigación social Recuperado de: http://www.insumisos.com/lecturasinsumisos/Epistemologia $\% 20$ es\%ciencias\%20sociales.pdf (citado 17 febrero 2009

Cabal O, Gutiérrez M, y Pazmiño A. (2016). Tu amigo de la "U”. (Trabajo de práctica). Universidad Nacional abierta y a distancia UNAD, Cali, Colombia. 
Climent Bonilla, J B; (2010). Algunas Aplicaciones De La Teoría De Sistemas Al Desarrollo

Organizacional. Revista Mexicana de Agronegocios, () 388-396. Recuperado de http://www.redalyc.org/articulo.oa?Id=14114743009

Consejo superior universitario Universidad Nacional Abierta y a Distancia (2012). Acuerdo 0015 del 30 de marzo de 2012. Por el cual se modifica el Estatuto general de la universidad Nacional Abierta y a Distancia (UNAD). Recuperado de https://sgeneral.unad.edu.co/images/documentos/consejoSuperior/acuerdos/2012/COSU_A CUE_0015_30032012.pdf

Desing Thinking (sf). ¿Qué es el Desing Thinking? Recuperado de: http://designthinking.es/inicio/index.php?utm_source=blog\&utm_medium=post\&utm_cam paign $=$ fromdoppler

Díaz, G \& Ortiz, R. (2005) La Entrevista Cualitativa. Universidad mesoamericana, cultura de investigación universitaria. Pp: 22- 27. Recuperado de http://www.geiumaoax.net/cursos/entrevistacualitativa.pdf

Díaz, L. (2010). La Observación. (1. Ed.). México: Universidad Nacional Autónoma de México, facultad de psicología. Recuperado de: http://www.psicologia.unam.mx/documentos/pdf/publicaciones/La_observacion_Lidia_Dia z_Sanjuan_Texto_Apoyo_Didactico_Metodo_Clinico_3_Sem.pdf

Duque Oliva, E J; (2005). Revisión del concepto de calidad del servicio y sus modelos de medición. INNOVAR. Revista de Ciencias Administrativas y Sociales, 15() 64-80. Recuperado de http://oai.redalyc.org/articulo.oa?id=81802505 
Elizalde, A., Martí, M. \& Martínez, F. (2006). Una revisión crítica del debate sobre las necesidades humanas desde el Enfoque Centrado en la Persona. Polis. Recuperado de http://www.redalyc.org/pdf/305/30517306006.pdf

Gómez D (2016). Los 5 niveles de servicio y la consecuente reacción del cliente. Recuperado de: http://bienpensado.com/5-niveles-de-servicio/

Gómez J. (2013). Grupos Focales: Herramienta y uso en la Investigación. Recuperado de: http://www.redalyc.org/articulo.oa?id=79830021014

Gómez, D. (2017, 5 de mayo). Qué hacer para enamorar a sus clientes. Emprende. Recuperado de http://mprende.co/marketing/\%C2\%BFqu\%C3\%A9-hacer-para-enamorar-sus-clientes

Hamui-Sutton, A; Varela-Ruiz, M; (2013). La técnica de grupos focales. Investigación en Educación Médica, 2() 55-60. Recuperado de http://revele.com.veywww.redalyc.org/articulo.oa?id=349733230009

Hartmann P. (2015). Cómo medir el impacto del servicio al cliente en la rentabilidad. Recuperado de: https://www.linkedin.com/pulse/c\%C3\%B3mo-medir-el-impacto-del-servicio-alcliente-en-la-pablo-hartmann

Horovitz, J. (1990). La calidad del servicio. Madrid: McGraw-Hill Interamericana. Recuperado de: http://www.scielo.org.co/pdf/inno/v15n25/v15n25a04.pdf

ISO (International Standard Organization) 9001 (2013). Sistemas de Gestión de Calidad según ISO 9000-2008. Recuperado de: http://iso9001calidad.com/definicion-de-terminos-586.html ISO (International Standard Organization) 9001 (2015). La UNAD obtiene nuevamente la recertificación del componente de gestión de la calidad 2015-2018. Recuperado de http://iso9001calidad.com/definicion-de-terminos-586.html 
Jiménez, X. (2003). Universidad de Cantabria. Teoría de la acción razonada. Recuperado en http://ocw.unican.es/ciencias-de-la-salud/ciencias-psicosociales-i/materiales/bloquetematico-iv/tema-14.-la-adherencia-al-tratamiento-1/14.4.2-teoria-de-la-accion-razonadaajzen-y

Ministerio de educación (2015). Guía para la Implementación de Educación Superior del Modelo de Gestión de Permanencia y Graduación Estudiantil en Instituciones de Educación Superior. Recuperado de: http://www.mineducacion.gov.co/1759/articles356272_recurso.pdf

Ministerio de Educación Nacional (2006). Decreto Número 2770 del 16 de agosto 2006 "Por el cual se transforma en ente autónomo a la universidad Nacional Abierta y a Distancia UNAD-, y se dictan otras disposiciones" Recuperado de: https://informacion.unad.edu.co/images/acerca\%20de\%20la\%20unad/gobierno\%20corporat ivo/Decreto_2770_2006.pdf

Mora Vargas, A I; (2005). Guía para elaborar una propuesta de investigación. Educación, 29() 6797. Recuperado de http://www.redalyc.org/articulo.oa?id=44029206

Ojeda, M. (2016). La condensación de historias en el storytelling publicitario: análisis de la campaña Cholo Soy de Mi banco (Tesis de pregrado en Comunicación). Universidad de Piura. Facultad de Comunicación. Piura, Perú.

Reichheld F. (2006) The Ultimate Question, Harvard Business School Press, recuperado de: http://www.jihel.be/wp-content/uploads/2006/02/Ultimate_question_excerpt.pdf

Samaja, J. (1994). Epistemología y Metodología. Buenos Aires. EUDEBA. Recuperado de: http://www.redalyc.org/pdf/184/18415426004.pdf 
Taylor, S.J. y Bogdan, R. (1986). Introducción a los métodos cualitativos de investigación. La búsqueda de significados. Buenos Aires: Editorial Paidós. Recuperado de: http://www.terras.edu.ar/aula/cursos/10/biblio/10TAYLOR-S-J-BOGDAN-R-Metodologiacualitativa.pdf

Tomat, C; (2012). El "Focus group": nuevo potencial de aplicación en el estudio de la acústica urbana. Athenea Digital. Revista de Pensamiento e Investigación Social, 12() 129-152. Recuperado de http://www.redalyc.org/articulo.oa?id=53723279006

Torres, Luz (2012). Retención estudiantil en la Educación Superior. Bogotá: Editorial Pontificia Universidad Javeriana.

Universidad de los Andes (2015). Diseño y gestión de la experiencia del cliente. Recuperado de https://administracion.uniandes.edu.co/phocadownload/programasabiertos/2015/03/12/diseo\%20y\%20gestin $\% 20 \mathrm{de} \% 201 \mathrm{a} \% 20$ experiencia $\% 20 \mathrm{del} \% 20 \mathrm{cliente}$. pdf

Velásquez, A. (2007, septiembre-diciembre). La Organización, el sistema y su dinámica: Una versión de Niklas Luhmann. Revista Escuela de Administración de Negocios, núm. 61, pp. 129-155.

Vicerrectoría de servicios a aspirantes, estudiantes y egresados (2015). Caracterización del estudiante Unadista.

Villarroel, G; Brito Alfonso, M; Armas, E d; (2004). Representaciones sobre la libertad y la igualdad en estudiantes venezolanos. Revista Venezolana de Economía y Ciencias Sociales, 10() 181-193. Recuperado de http://www.redalyc.org/articulo.oa?id=17710213 